\title{
HARPO, a gaseous TPC as a gamma telescope and polarimeter: first measurement in a polarised photon beam between 1.7 and $74 \mathrm{MeV}$
}

\section{A. Delbart*}

Irfu, CEA-Saclay, Gif-sur-Yvette, France

E-mail: alain.delbartecea.fr

\author{
S. Amano ${ }^{b}$, D. Attié ${ }^{a}$, D. Bernard ${ }^{c}$, P. Bruel ${ }^{c}$, D. Calvet $^{a}$, P. Colas ${ }^{a}$, S. Daté ${ }^{d}$,

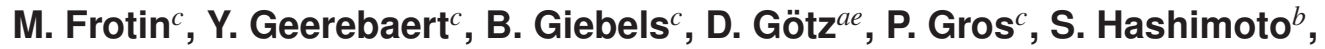 \\ D. Horan ${ }^{c}$, T. Kotaka ${ }^{b}$, M. Louzir ${ }^{c}$, Y. Minamiyama ${ }^{b}$, S. Miyamoto ${ }^{b}$, H. Ohkuma ${ }^{d}$,

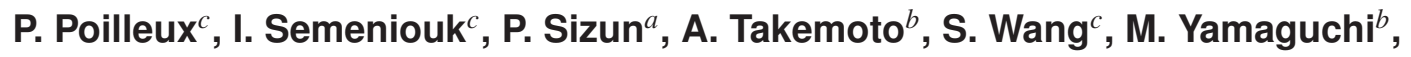 \\ ${ }^{a}$ Irfu, CEA-Saclay, Gif-sur-Yvette, France \\ ${ }^{b}$ LASTI, University of Hyôgo, Japan \\ ${ }^{c}$ LLR, Ecole Polytechnique, CNRS/IN2P3, Palaiseau, France \\ d JASRI/SPring8, Japan \\ ${ }^{e}$ AIM, CEA/DSM-CNRS-Université Paris Diderot, France
}

\begin{abstract}
Gamma-ray astronomy enables the exploration of the non-thermal emission and magnetic field configuration of objects such as active galactic nuclei (AGN), gamma ray bursts (GRB) and pulsars. Presently, there is a sensitivity gap between $1 \mathrm{MeV}$ and $100 \mathrm{MeV}$. Additionally, there is no polarisation measurement above $1 \mathrm{MeV}$, although such a measurement could shed light on emission processes. A gaseous detector can achieve a much better angular resolution in the $\mathrm{MeV}-\mathrm{GeV}$ range than the current/past telescopes that use tungsten converters, thanks to the reduced multiple scattering of the electrons and positrons. This translates to a greatly improved point source sensitivity and gives access to the linear polarisation of the photons through the azimuthal angle of the pair.

The HARPO time projection chamber (TPC) is a high angular resolution telescope for gamma-ray polarimetry. It was installed in a polarised gamma-ray beam at NewSUBARU in Japan in 2014. Data were taken at photon energies from $1.7 \mathrm{MeV}$ to $74 \mathrm{MeV}$, and with different polarisation configurations. The experimental setup of the TPC and the photon beam are described. The first results from the beam campaign are shown.
\end{abstract}

The 34th International Cosmic Ray Conference,

30 July- 6 August, 2015

The Hague, The Netherlands

\footnotetext{
* Speaker.
} 


\section{Science case}

High-energy photons such as $\gamma$-rays emitted by cosmic sources are produced by non-thermal phenomena that involve high-energy charged particles. They provide unique information on the processes at work in objects such as active galactic nuclei (AGN), pulsars and gamma-ray bursts (GRB). Presently $\gamma$-ray astronomy suffers from a gap in sensitivity between the energy ranges in which Compton telescopes (sub-MeV), and $e^{+} e^{-}$-pair telescopes (above $1 \mathrm{GeV}$ ) excel. In principle, pair telescopes could be sensitive down to threshold, but in practice the bad angular resolution at low energy hinders their ability to assign a given photon to a particular source. This situation is detrimental to the understanding of objects that have a multi-peak spectral energy distribution (SED), such as AGN, and to objects whose emission is peaking in the $\mathrm{MeV}$ region (the prompt emission of many GRB). A better sensitivity is also needed to track high-energy cosmic-rays from super-nova remnants (SNR) from their hadronic interactions, by the detection of the $\pi^{0}$ bump at $\approx 100 \mathrm{MeV}$. An improved angular resolution is needed to observe crowded regions of the $\gamma$-ray sky, such as in the centre of our galaxy.

At lower photon energies, from radio-waves to soft-X-rays, polarimetry provides a powerful insight to phenomena at work inside cosmic sources, in particular it provides a means to measure the magnetic field. Above a couple of $\mathrm{MeV}$, this diagnostic is missing: not only does the Compton cross section decrease at high energy, but its polarisation asymmetry also decreases like $1 / E$ [1]. Polarimetry would allow, for example, to decipher leptonic from hadronic models of blazars [2]. In the case of a $\gamma$-ray production by synchrotron emission of high-energy protons, the linear polarisation fraction stays high from X-ray to $\gamma$-ray energies. In the case of "leptonic" emission though, $\mathrm{X}$-ray emission is highly polarised, but the $\gamma$-ray part of the spectrum is completely washed out by the inverse Compton scattering of thermal photons, and the total emission is unpolarised. Polarimetry is also of interest for searches of Lorentz invariance violation (LIV) using GRBs [3]. In the presence of LIV, the induced birefringence of the vacuum would result in an energy-dependent rotation of the polarisation direction of a (supposedly) initially polarised emission, upon propagation. On average this would result in an unpolarised radiation: if a non-zero polarisation of distant GRB is observed, an upper limit on LIV-producing effects by yet-unknown physics beyond the standard model is derived [3]. As the rotation angle is proportional to the squared photon energy, extending polarimetry to the $\gamma$-ray energy range would enable a huge gain in sensitivity.

\section{Telescope and polarimeter with a thin homogeneous detector and an optimal tracking}

Past and presently active pair telescopes use an active target that consists of a "mille-feuille", a multi-layer set of alternatively high- $Z$ converter slabs and sensor foils. In the case of the $\mathrm{Si} / \mathrm{W}$ tracker of the Fermi/LAT, the single photon angular resolution is dominated by the multiple scattering of the electrons in the slabs and scales like $E^{-0.78}$ below $10 \mathrm{GeV}$, and amounts to $\approx 4^{\circ}$ at $100 \mathrm{MeV}$ [4]. Doing better with this technology would involve thinner and/or lower- $Z$ converters, a severe issue for space missions for which the number of electronic channels is limited. A way out is the use of a low-density (here gas) detector such as a time projection chamber (TPC), a detector type widely used in particle physics: a volume of (here homogeneous) material is immersed in a 
(here uniform) electric field. Upon occurrence of an "event", that is of the conversion of a photon to an $e^{+} e^{-}$pair, the two tracks ionise the gas after which the ionisation electrons drift towards an anode where they are amplified, collected and sampled in time. A segmented anode provides a time "map" of the signal over the two directions $x$ and $y$ transverse to the electric field. Time sampling provides a measurement of the drift duration, that is of the drift length $z$. In total a 3D image of the event is provided at a reasonable cost in terms of number of electronics channels. The single-photon angular resolution includes several contributions:

- Recoil. In the case of a "nuclear" conversion, that is a conversion by interaction of the photon with the field of a nucleus of the detector, the energy that is transferred to the nucleus is small and the track length is too short to be reconstructed. The contribution of the recoil momentum to the reconstructed momentum of the photon is therefore missing. Given the high-momentum tail of the recoil momentum distribution (Fig. 3 of [5]), we have estimated the contribution to the angular resolution by the angle that includes $68 \%$ of the events [5] to be $\approx 1.5 \operatorname{rad}(E / 1 \mathrm{MeV})^{-5 / 4}$.

Triplets. In the case of the conversion in the field of an electron of the detector, the track of the recoiling electron can be long enough that it can be reconstructed, in which case its contribution can be taken into account in the reconstruction: the event is then called a "triplet", as three tracks are visible in the final state. Unfortunately these events are too few to contribute significantly to the differential sensitivity (slide 20 of [6]). As the azimuthal angle of the recoil is correlated with the direction of polarisation of the incident photon, hope has raised to perform polarimetry with triplet ([7] and references therein). Alas, the cross section above a threshold recoil momentum gets low at low photon energy (Fig. 6 of [8]), and in total the potential of triplet for polarimetry is quite limited (section 5.3 of [8]).
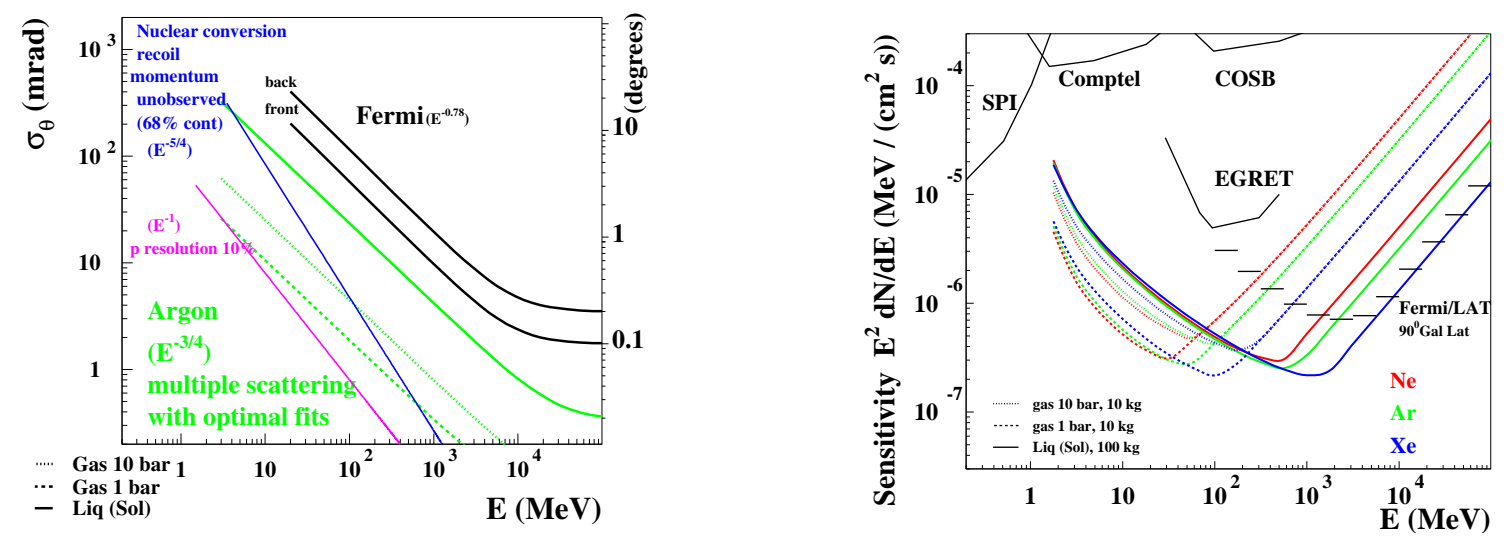

Figure 1: (Colour online). Left: various contributions to the photon angular resolution; Right: point-like source differential sensitivity [5].

- Next comes the multiple scattering of the tracks in the TPC material. These angular deflections induce correlated deviations between successive position measurements of a track, that must be taken into account in the reconstruction. It can be shown that this can be performed in an optimal way by a Kalman-filter-based fitter [9]. In that case, in the continuous detector 
limit [10], the expression of the RMS track angular resolution at its starting point is $[5,8]$ $\sigma_{\theta t L}=\left(p / p_{1}\right)^{-3 / 4}$, where $p$ is the track momentum, $p_{1} \equiv p_{0}\left(4 \sigma^{2} l / X_{0}^{3}\right)^{1 / 6}$ is a characteristic momentum that parametrises the performance of the tracking under multiple-scattering of a given detector, and $p_{0}=13.6 \mathrm{MeV} / c$ is the constant that appears in the expression of multiple scattering (eq. (32.15) of [11]). In the expression for $p_{1}, \sigma$ is the single-point spatial resolution, $l$ is the track longitudinal sampling, and $X_{0}$ is the radiation length of the tracker material. The single-track angular resolution is extended easily to the average single-photon angular resolution by multiplication by a factor that is close to unity [5]. The $p^{-3 / 4}$ variation compares nicely with the experimental value of $E^{-0.78}$ for the Fermi/LAT collaboration [4]. For $l=1 \mathrm{~mm}, \sigma=0.1 \mathrm{~mm}$ and a 10 bar argon-based gas, $\sigma_{\theta t L}=0.26^{\circ}$ at $E=100 \mathrm{MeV}$, while the recoil contribution amounts to $0.27^{\circ}$ (Fig. 1), The two curves cross close to $100 \mathrm{MeV}$, and the total angular resolution amounts to $0.4^{\circ}$ at $100 \mathrm{MeV}$, which is an order of magnitude improvement with respect to the Fermi/LAT. The expression for $\sigma_{\theta t L}$ was cross-checked with an actual Kalman-filter (Fig. 18 of [8]).

- Detector resolution. At the highest energy, the angular resolution flattens to an asymptote dominated by the detector resolution (Fig. 1 left).

- Momentum resolution. Track momentum can be measured either directly in the TPC, if immersed into a magnetic field, or with additional external detectors such as a transition radiation detector (TRD) or a calorimeter. For the lowest energies, the momentum can be estimated by the multiple measurement of multiple scattering in the TPC alone ([5] and references therein). For a $10 \%$ resolution, the contribution to the photon angular resolution is negligible (Fig. 1 left).

The improvement in angular resolution translates to an improvement in point-like source differential sensitivity, as is shown in Fig. 1 right. Indeed, together with the high-performance Comptontelescope projects presently under development, closing the sensitivity gap is within reach.

Polarimetry has long be considered to be out of reach for $e^{+} e^{-}$-pair telescopes as multiple scattering blurs the azimutal angular information, that between the conversion plane and a direction transverse to the propagation of the photon. Following the approximation of [12], that is an opening angle between the $e^{+} e^{-}$pair equal to its most probable value, the resolution for the azimuthal angle is independent of photon-energy. In a mille-feuille telescope, after a path length of $\approx 10^{-3} X_{0}$ in the conversion slab, the polarisation asymmetry already undergoes a dilution of the polarisation asymmetry of a factor of two [8]. In a homogeneous detector though, the resolution for the azimuthal angle varies like $E^{1 / 4}$ (eq. (18) of [8]): there is hope that in the lower part of the energy spectrum of a pair telescope, where most of the statistics lies, the dilution stays high ${ }^{1}$. We have written an event generator which samples from the full, 5D, exact down to threshold (without any high-energy approximation) differential cross section, and includes polarisation [8]. We have used it to study the performance of a homogeneous tracker with optimal tracking as described above, using the full 5D event probability density function (pdf). We found that for a gas TPC the dilution stays indeed very close to unity over most of the energy spectrum (Fig. 20 of [8]). With

\footnotetext{
${ }^{1}$ We are using the high-energy physics definition of the dilution $D$ of the asymmetry $\mathscr{A}$, that is $D \equiv \mathscr{A}_{\text {eff }} / \mathscr{A}_{\text {, that is }}$ the higher the value of $D$, the better.
} 
a $1 \mathrm{~m}^{3} 5$ bar argon TPC, one full (exposure fraction of $\eta=1$ ) year exposure to a Crab-like source and with experimental cuts applied, a $P$ precision of $1.4 \%$ would be reached [8].

\section{HARPO: the detector}

The HARPO demonstrator is a $30 \mathrm{~cm}$ cubic TPC surrounded by 6 scintillator plates equipped with two wavelength shifter bars and two photomultipliers (PMTs) for trigger and background rejection. The gas container vessel is an aluminum cylinder designed to be operated from few mbar up to 5 bar in sealed mode (Fig. 2). The TPC is filled with a mixture of $95 \%$ of argon and $5 \%$ of $\mathrm{iC}_{4} \mathrm{H}_{10}$ and operated at a $220 \mathrm{~V} / \mathrm{cm}$ drift electric field. The TPC end-plate is a "hybrid" MicroPattern Gaseous Detector (MPGD) which is composed of two GEM foils[13] stacked with $2 \mathrm{~mm}$ spacing above a $128 \mu \mathrm{m}$ amplification gap bulk-micromegas[14]. The anode collection plane is segmented in 2 times 288 strips in the two orthogonal $x-y$ directions read by an electronic chain composed of two T2K/TPC Front-End cards, two FEMINOS back-end cards and a Trigger Clock Module [15]. An innovative trigger system, called FAST (Fully Automated Switched Trigger), makes use of the FPGA of a modified PMm2 trigger card to generate up to 16 trigger lines built with combinations of the signals from the 12 PMTs, micromegas mesh signal, and an external laser trigger. This demonstrator was extensively studied in laboratory with a ${ }^{55} \mathrm{Fe} 5.9 \mathrm{keV} \mathrm{X}$-ray source and with cosmic rays to measure its performances (MPGD gas gain absolute calibration, optimisation and stability, track reconstruction, electronic noise, electron attachment, electron drift velocity) and determine its operating conditions (MPGD high voltages, readout electronics dynamic range and sampling frequency, trigger configuration) [16].
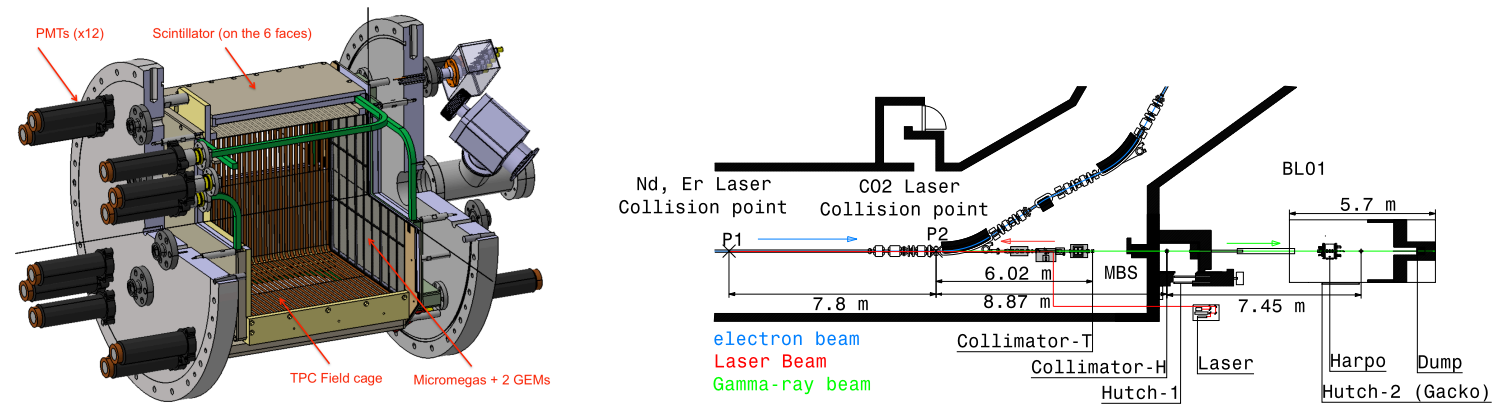

Figure 2: Left: a 3D CAD drawing of the HARPO demonstrator; Right: the gamma beam line and test setup at NewSUBARU.

\section{Detector test with a polarised gamma-ray beam}

In November 2014, the HARPO demonstrator was exposed to a pseudo-monochromatic and almost fully polarised $\gamma$-ray beam delivered by the BL01 beam line of the NewSUBARU storage ring [17] (figure 2) which is operated by the Laboratory of Advanced Science and Technology for Industry (LASTI) of the University of Hyôgo (Japan). The $\gamma$-ray beam is produced by the inverse Compton scattering of laser photons on the relativistic electrons stored in NewSUBARU (Laser Compton Source or LCS). We used four electron beam energies: 0.618, 0.982, 
1.223 and $1.480 \mathrm{GeV}$. We could cover the $\gamma$-ray energy range from $1.74 \mathrm{MeV}$ up to $74.3 \mathrm{MeV}$ through four available laser photon wavelengths: $\lambda=0.532 \mu \mathrm{m}$ and $\lambda=1.064 \mu \mathrm{m}$ with a $20 \mathrm{kHz}$ pulsed Nd: $\mathrm{YVO}_{4}(2 \omega$ and $1 \omega)$ laser, $\lambda=1.540 \mu \mathrm{m}$ with a $200 \mathrm{kHz}$ pulsed Er (fibre) laser and $\lambda=10.55 \mu \mathrm{m}$ with a CW CO 2 laser (Fig. 5). In order to select the Compton energy edge, we used two $100 \mathrm{~mm}$ long collimators made of lead bricks and which we precisely aligned on the beam axis by $\gamma$-ray energy measurements with a $\mathrm{NaI}$ detector. When collimation is applied, the laser beam linear polarisation (measured to be above 99.9\%) is almost fully transferred to the $\gamma$-ray beam. For systematic bias studies, the laser beam was also changed to circular polarisation by use of a quarter-wave plate, which, for an experiment that is not sensitive to the polarisation of the leptons, is equivalent to random polarisation $(P=0)$.

The HARPO demonstrator was filled with gas at 2 bar after successive vacuum pumpings and rinsings. The main effects of gas degradation are a modification of the amplification gain and a loss of signal due to capture of electrons along the drift. Figure 3 shows a measure of the relative gain and electron drift velocity over the two weeks of data taking, with a slight variation of gain and drift velocity (around 5\%). A monitoring of the temperature and pressure shows that we had a very low leakage rate.

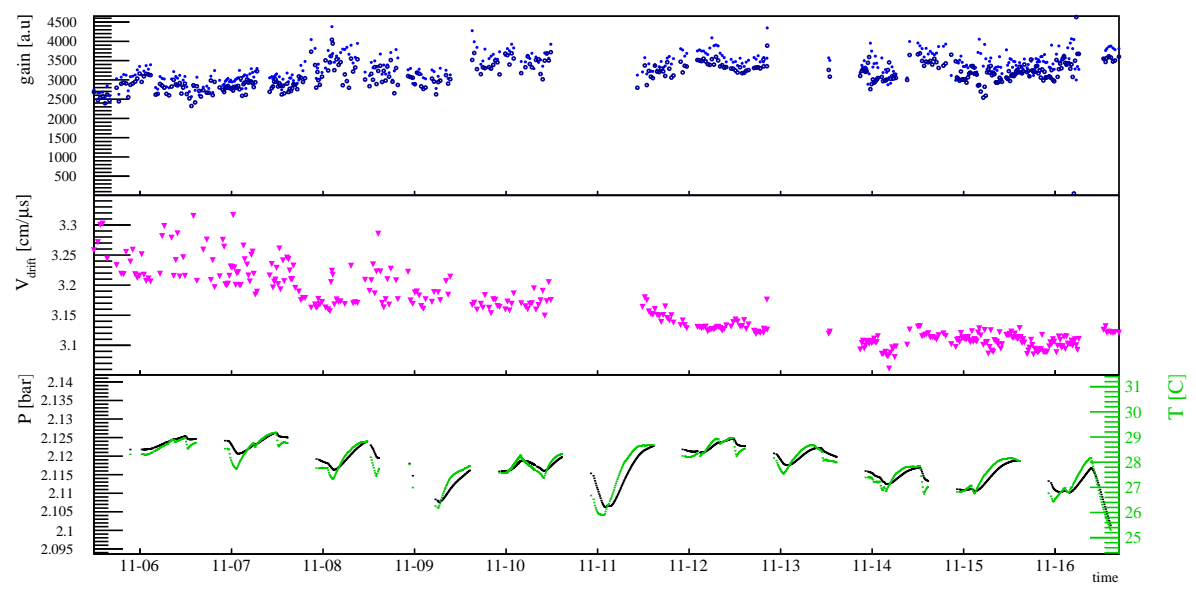

Figure 3: Evolution of the gain, electron drift velocity, temperature and pressure during data taking.

The main trigger line selects $\gamma$-ray from LCS conversion in the gas. It uses the signal of the scintillators, the start signal from the pulsed laser, and the signal from the micromegas mesh. It was designed to select events with conversion in the TPC gas, while rejecting conversions in the upstream material. This was done by applying a veto on "early" signals in the mesh, which come from electrons with zero or short drift. Additional trigger lines were used to check the efficiency of the different trigger elements. Figure 4 shows a selection of conversion events. The main raw output is a pair of 2D maps, representing the $x-z$ and $y-z$ projections, where $z$ is obtained from the arrival time of the electrons. In most cases, we see nuclear conversion, where only the $e^{+} e^{-}$pair is visible. Occasionally, we see triplet conversions, where the recoil electron forms a third track. An estimation of the rate of gamma conversions in the gas comes from looking at the position of the "start'" (i.e. $z / t$ minimum, the point closest to the beam side) of an event. This point is an approximation of the vertex position (Fig. 4). If that point is within the transverse geometrical 


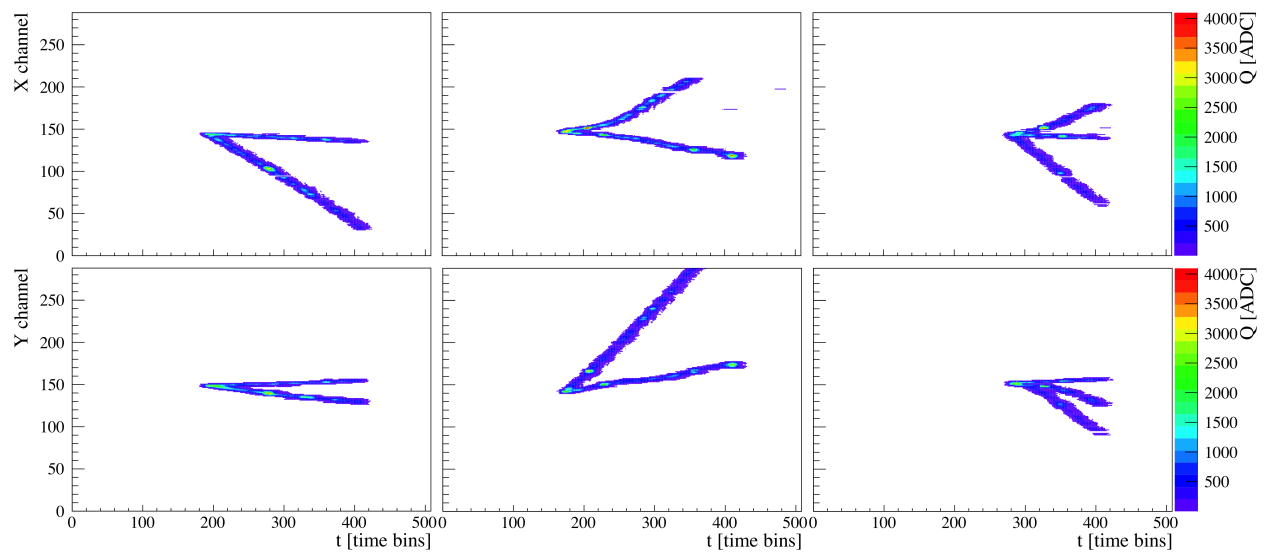

Figure 4: Raw data from 3 pair conversion events. Left : a 74.3 MeV photon; Middle : a $4.68 \mathrm{MeV}$ photon; Right : a $74.3 \mathrm{MeV}$ photon with recoil electron (triplet conversion). The top and bottom represent the $x-z$ and $y-z$ projections. The signal is spread out by diffusion and electronics shaping, which are taken into account in the reconstruction algorithms. 1 time bin $=30 \mathrm{~ns}$ and $1 \mathrm{ADC}$ count $\approx 0.03 \mathrm{fC}$.

location of the $\gamma$-ray beam and longitudinal location of the TPC gas, we estimate that the event corresponds to an interaction of the beam with the gas. Figure 5 shows how this selection is done, and gives the average fraction of events with a $\gamma$-ray conversion in the gas. The acquisition rate reached up to $50 \mathrm{~Hz}$ for a total of nearly 60 million events, of which more than 30 million are due to an interaction of the photons with the gas. At the considered energies, most of these events should be pair conversions.
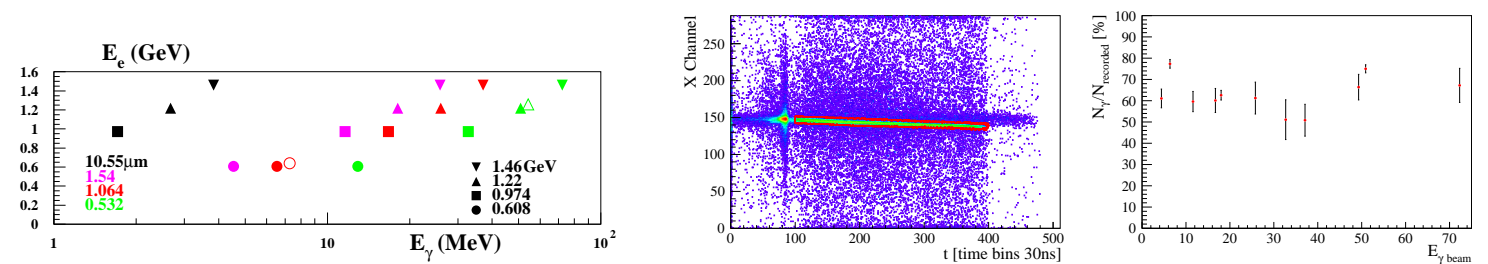

Figure 5: Left: $\gamma$ energies as a function of electron energies and laser wavelengths; Middle: distribution of the position of the first signal in the TPC for each event in one run. The central region (in red) represents the beam position. Events in that region are considered to contain an interaction of the $\gamma$-rays with the gas. Right: resulting fraction of all recorded events which contain $\gamma$-rays for most of the photon energies used.

\section{Conclusion and Prospectives}

The HARPO TPC demonstrator was exposed to polarised gamma photons with energies between $1.74 \mathrm{MeV}$ and $74.3 \mathrm{MeV}$. The data taking was efficient with more than $50 \%$ of high quality events coming from gamma conversion to $\mathrm{e}^{+} \mathrm{e}^{-}$pairs inside the TPC gas volume. A complete reconstruction and characterisation of these events is on-going to measure the angular resolution of the detector, as well as its sensitivity to the photon polarisation. In the perspective of a stratospheric 
balloon flight test, several R\&D programs are on-going on the design of a rad-hard electronics, the optimization of the MPGD by use of a thin gap micromegas optimised for high pressure, and the development of an advanced trigger and a gas recirculation/purification system.

This work is funded by the French National Research Agency (ANR-13-BS05-0002) and was performed by using NewSUBARU-GACKO (Gamma Collaboration Hutch of Konan University).

\section{References}

[1] D. Bernard, Compton polarimetry revisited, arXiv:1507.02824 [astro-ph.IM].

[2] H. Zhang and M. Böttcher, X-Ray and Gamma-Ray Polarization in Leptonic and Hadronic Jet Models of Blazars, Astrophys. J. 774, 18 (2013) [arXiv:1307.4187 [astro-ph.HE]].

[3] D. Götz et al., GRB 140206A: the most distant polarized Gamma-Ray Burst, Mon. Not. Roy. Astron. Soc. 444 (2014) 3, 2776 [arXiv:1408.4121 [astro-ph.HE]].

[4] M. Ackermann et al., The Fermi Large Area Telescope On Orbit: Event Classification, Instrument Response Functions, and Calibration, ApJS 203 (2012) 4, [arXiv:1206.1896 [astro-ph.IM]].

[5] D. Bernard, TPC in gamma-ray astronomy above pair-creation threshold, Nucl. Instrum. Meth. A 701 (2013) 225 [Nucl. Instrum. Meth. A 713 (2013) 76] [arXiv:1211.1534 [astro-ph.IM]].

[6] D. Bernard, A thin detector as a high angular resolution gamma ray telescope (and polarimeter) in the MeV-TeV energy range; Towards a post-Fermi mission? Gamma2012, 5th International Symposium on High-Energy Gamma-Ray Astronomy, Heidelberg, July 9-13 2012.

[7] V. F. Boldyshev et al., Measurement of linear polarization of photons by using the asymmetry of recoil electrons in the photoproduction of triplets, Phys. Atom. Nucl. 58 (1995) 39 [Yad. Fiz. 58 (1995) 43].

[8] D. Bernard, Polarimetry of cosmic gamma-ray sources above $e^{+} e^{-}$pair creation threshold, Nucl. Instrum. Meth. A 729 (2013) 765 [arXiv:1307.3892 [astro-ph.IM]].

[9] R. Frühwirth, Application of Kalman filtering to track and vertex fitting, Nucl. Instrum. Meth. A 262, 444 (1987).

[10] W. R. Innes, Some formulas for estimating tracking errors, Nucl. Instrum. Meth. A 329, 238 (1993).

[11] K. A. Olive et al. [Particle Data Group Collaboration], Review of Particle Physics, Chin. Phys. C 38 (2014) 090001.

[12] Yu. D. Kotov, Methods of measurement of gamma-ray polarization, Space Sci. Rev. 49 (1988) 185.

[13] F. Sauli, GEM: A new concept for electron amplification in gas detectors, Nucl. Instrum. Meth. A 386, (1997) 531.

[14] I. Giomataris et al., Micromegas in a bulk, Nucl. Instrum. Meth. A 560, (2006) 405.

[15] D. Calvet, A Versatile Readout System for Small to Medium Scale Gaseous and Silicon Detectors, IEEE Trans. Nucl. Sci. 61 (2014) 675.

[16] S. Wang et al., HARPO: beam characterization of a TPC for gamma-ray polarimetry and high angular-resolution astronomy in the $\mathrm{MeV}-\mathrm{GeV}$ range, [arXiv:1503.03772 [astro-ph.IM]].

[17] S. Amano et al., Several MeV $\gamma$-ray generation at NewSUBARU by laser Compton backscattering, Nucl. Instrum. Meth. A 602 (2009) 337. 